\title{
Obesity-Induced Inflammation Cooperates with Loss of DNA Methyltransferase 3A to Develop Early-Onset of Leukemia
}

Taruni Reddy Pandhiri ${ }^{1, \#}$, Santhosh Kumar Pasupuleti ${ }^{2}$, Baskar Ramdas $^{2}$, Rahul Kanumuri², Reuben Kapur ${ }^{2,3, *}$

${ }^{1}$ Indiana University School of Medicine, Indianapolis, Indiana, USA; ${ }^{2}$ Herman B Wells Center for Pediatric Research; ${ }^{2}$ Department of Microbiology and Immunology; ${ }^{3}$ Department of Medical and Molecular Genetics, Indiana University School of Medicine, Indianapolis, Indiana, USA.

*Corresponding author: rkapur@iupui.edu

\#Presenting author: tarpandh@iu.edu

Obesity is an increasing epidemic disease world-wide responsible for enhancing the risk for developing Type 2 diabetes mellitus (T2DM) as well as cancer. However, it is unclear if and how obesity contributes to the transformation of pre-leukemic stem and progenitors (pre-LHSC/Ps) into full-blown leukemia such as acute myeloid leukemia (AML) or severe form of myeloproliferative neoplasm (MPN). We hypothesized that obesity induced chronic inflammation might be responsible for clonal selection of pre-LHSC/Ps bearing pre-leukemic mutations such as DNA methyltransferase $3 \mathrm{~A}$ (DNMT3A) and for promoting the progression of early-onset MPN towards severe forms of AML/leukemia. To test this hypothesis, we genetically crossed preleukemic $D n m t 3 a^{+/-} ; M x-C r e+$ mice with leptin deficient obese $\left(L e p^{O b / O b}\right)$ mice to obtain $\mathrm{Ob} / \mathrm{Ob} ; \mathrm{Dnmt}_{3} \mathrm{a}^{+/-} ; \mathrm{Mx}-\mathrm{Cre}+$ compound mutant mice. Further, the Dnmt3a gene was deleted by giving the PolyIC and the deletion was confirmed through PCR. After 12 days of post-PolylC the myeloid cells (neutrophils and monocytes) were expanded in $\mathrm{Ob} / \mathrm{Ob} ; \mathrm{Dnmt} 3 \mathrm{a}^{+/-}$;Mx-Cre+ mice compared to Dnmt3a ${ }^{+/} ; M x-C r e+, D n m t 3 a^{+/-} ; M x-C r e-, O b / O b$ and $W T$ mice. We have harvested and analyzed all these mice after 26 days of post-PolylC. Interestingly, Ob/Ob;Dnmt3a ${ }^{+/-} ; M x-$ Cre+ mice showed increased BM cellularity, both the frequency of lineage negative, Sca-1+ and c-KIT+ (LSK) cells, short-term hematopoietic stem cells (ST-HSCs; LSK/CD48+/CD150-), granulocyte macrophage progenitor (GMPs; LSK/CD16+/CD34+), and reduction in LT-HSCs (LT-HSCs; LSK/CD48-/CD150+) compared to other groups. Flow cytometry analysis of PB, BM and spleen from $\mathrm{Ob} / \mathrm{Ob} ; \mathrm{Dnmt} 3 \mathrm{a}^{+/-} ; \mathrm{Mx}-\mathrm{Cre}+$ mice demonstrated a significant increase in the frequency of mature myeloid cells ( $\mathrm{Gr}-1+/ \mathrm{Mac}-1+)$ and a profound reduction in $\mathrm{B} 220+\mathrm{B}$ cells compared to other groups. Remarkably, these mice also showed splenomegaly, elevated heart size and early signs of AML blasts as reflected by the presence of $c-K I T+/ C D 11 b+$ double positive cells in the BM, consistent with severe MPN/AML development. Taken together, these results demonstrate that obesity induced inflammation cooperates with pre-leukemic $\mathrm{Dnmt} \mathrm{a}^{+/-}$ mutation to induce an early-onset of severe MPN/AML like disease. 